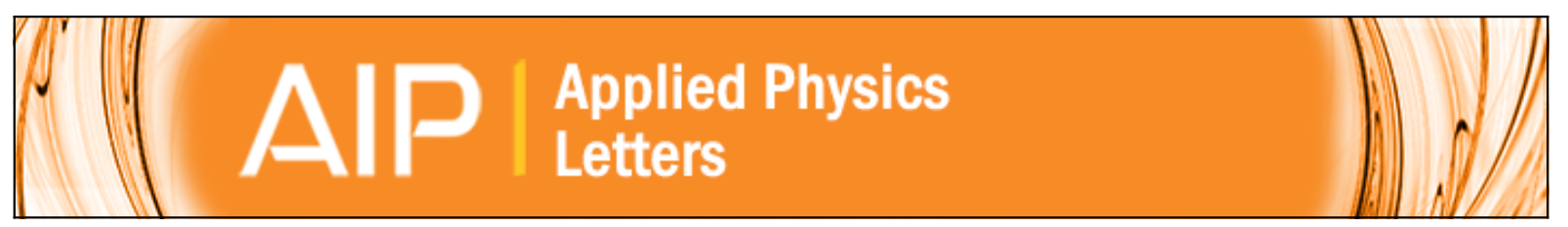

Atomic-scale observation of parallel development of super elasticity and reversible plasticity in GaAs nanowires

Peite Bao, Yanbo Wang, Xiangyuan Cui, Qiang Gao, Hung-Wei Yen, Hongwei Liu, Wai Kong Yeoh, Xiaozhou Liao, Sichao Du, H. Hoe Tan, Chennupati Jagadish, Jin Zou, Simon P. Ringer, and Rongkun Zheng

Citation: Applied Physics Letters 104, 021904 (2014); doi: 10.1063/1.4861846

View online: http://dx.doi.org/10.1063/1.4861846

View Table of Contents: http://scitation.aip.org/content/aip/journal/apl/104/2?ver=pdfcov

Published by the AIP Publishing 


\title{
Atomic-scale observation of parallel development of super elasticity and reversible plasticity in GaAs nanowires
}

\author{
Peite Bao, ${ }^{1}$ Yanbo Wang, ${ }^{2}$ Xiangyuan Cui,,${ }^{2,3}$ Qiang Gao, ${ }^{4}$ Hung-Wei Yen, ${ }^{2,3}$ Hongwei Liu, ${ }^{3}$ \\ Wai Kong Yeoh, ${ }^{2,3}$ Xiaozhou Liao, ${ }^{2, a)}$ Sichao Du, ${ }^{1} \mathrm{H}$. Hoe Tan, ${ }^{4}$ Chennupati Jagadish, ${ }^{4}$ \\ Jin Zou, ${ }^{5}$ Simon P. Ringer, ${ }^{2,3}$ and Rongkun Zheng ${ }^{1, b)}$ \\ ${ }^{1}$ School of Physics, The University of Sydney, Sydney, NSW 2006, Australia \\ ${ }^{2}$ School of Aerospace, Mechanical and Mechatronic Engineering, The University of Sydney, Sydney, \\ NSW 2006, Australia \\ ${ }^{3}$ Australian Centre for Microscopy \& Microanalysis, The University of Sydney, Sydney, NSW 2006, Australia \\ ${ }^{4}$ Department of Electronic Materials Engineering, Research School of Physics and Engineering, \\ The Australian National University, Canberra, ACT 0200, Australia \\ ${ }^{5}$ Materials Engineering and Centre for Microscopy and Microanalysis, The University of Queensland, \\ Brisbane, QLD 4072, Australia
}

(Received 5 December 2013; accepted 27 December 2013; published online 14 January 2014)

\begin{abstract}
We report the atomic-scale observation of parallel development of super elasticity and reversible dislocation-based plasticity from an early stage of bending deformation until fracture in GaAs nanowires. While this phenomenon is in sharp contrast to the textbook knowledge, it is expected to occur widely in nanostructures. This work indicates that the super recoverable deformation in nanomaterials is not simple elastic or reversible plastic deformation in nature, but the coupling of both. (C) 2014 AIP Publishing LLC. [http://dx.doi.org/10.1063/1.4861846]
\end{abstract}

Conventional theory and experiments in plasticity demonstrate that when a load is applied to metals and ceramics, they undergo first elastic deformation, followed by plastic deformation, and finally fracture. ${ }^{1}$ Elastic deformation is selfrecoverable and mediated by stretching of the bonds, but where the atoms do not slip past each other. In contrast, plastic deformation is usually irrecoverable or permanent and is mediated by discrete dislocation activity, displacive deformation twinning, stress-induced phase transformations, or fracture associated with flaws. ${ }^{2-4}$ Furthermore, it is well known that in ceramic crystals such as GaAs, the directional nature of the bonding makes dislocation movement and therefore plastic deformation very difficult. ${ }^{5}$ Therefore, bulk crystalline ceramic materials fracture after only a very small elastic strain of $\sim 0.1 \%$, and dislocation-mediated plastic strain is almost non-existent. ${ }^{6}$

On the other hand, nanowires (NWs) are known to support large elastic strains primarily because of the constrained size and number of grains across the NW diameter, which serves to greatly reduce the number of crystalline defects in the individual NWs and effectively prevents the initiation and propagation of cracks. ${ }^{7-9}$ Semiconductor NWs with recoverable strains of between $\sim 3 \%$ and $10 \%$ have been reported, and this strain is primarily elastic in nature. ${ }^{10-13}$ Recent research using computational simulations ${ }^{14,15}$ and nanoindentation experiments ${ }^{16,17}$ has revealed that nonlinear dislocation-mediated plasticity can also be reversible in some nanocrystalline materials.

To reveal the microscopic mechanisms of NWs deformation, atomic-scale characterization is indispensable. In this work, we developed an experimental method that enables atomic-scale microscopy of NWs under any particular level

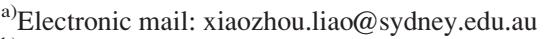

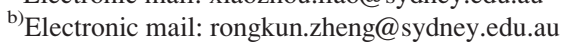

of bending deformation. We report the atomic-scale observation of parallel development of super elasticity and reversible plasticity throughout the whole range of bending deformation in GaAs nanowires. Although the dislocation density is extremely high, most of the plastic deformation spontaneously recovers upon the release of the external loading.

Twin-free GaAs NWs were grown by using metalorganic chemical vapor deposition (MOCVD) via the vaporliquid-solid mechanism using Au nanoparticles as catalyst. ${ }^{18}$ Trimethylgallium and $\mathrm{AsH}_{3}$ were used as the $\mathrm{Ga}$ and $\mathrm{As}$ sources, respectively. A two-temperature procedure, which consists an $1 \mathrm{~min}$ high-temperature $\left(450^{\circ} \mathrm{C}\right)$ nucleation step followed by a $30 \mathrm{~min}$ low-temperature $\left(375^{\circ} \mathrm{C}\right)$ growth step, was used and ensured samples were minimum tapered and of perfect crystalline quality 28 . NWs were vertically aligned on (111)B GaAs substrate and uniform in diameter of $\sim 50-60 \mathrm{~nm}$. NWs studied were $\sim 4-6 \mu \mathrm{m}$ in length.

High-resolution transmission electron microscopy (HRTEM) investigation was performed on a JEOL 2200FS field-emission TEM operating at $200 \mathrm{kV}$ and a JEOL $3000 \mathrm{~F}$ field-emission TEM operating at $300 \mathrm{kV}$ with a standard double-tilt mechanism. (111) d-spacing before, during, and after deformation are averaged values from three separate regions, each contains 15-20 lattices. Dislocation lines were identified via using inverse fast Fourier transformation (IFFT) of the HRTEM images. TEM sample preparation was done simply by scratching the growth substrate using copper grids with holey carbon supporting film.

Deformation experiments were performed in a Zeiss Auriga dual-beam scanning electron microscopy (SEM) equipped with a platinum gas injection system (GIS), and a Kleindiek Nanotechnik nanopositioning system connected with an electro-polished tungsten tip as the micromanipulator inside of the SEM chamber. GIS enables to affix the NW to the carbon supporting film by depositing platinum 

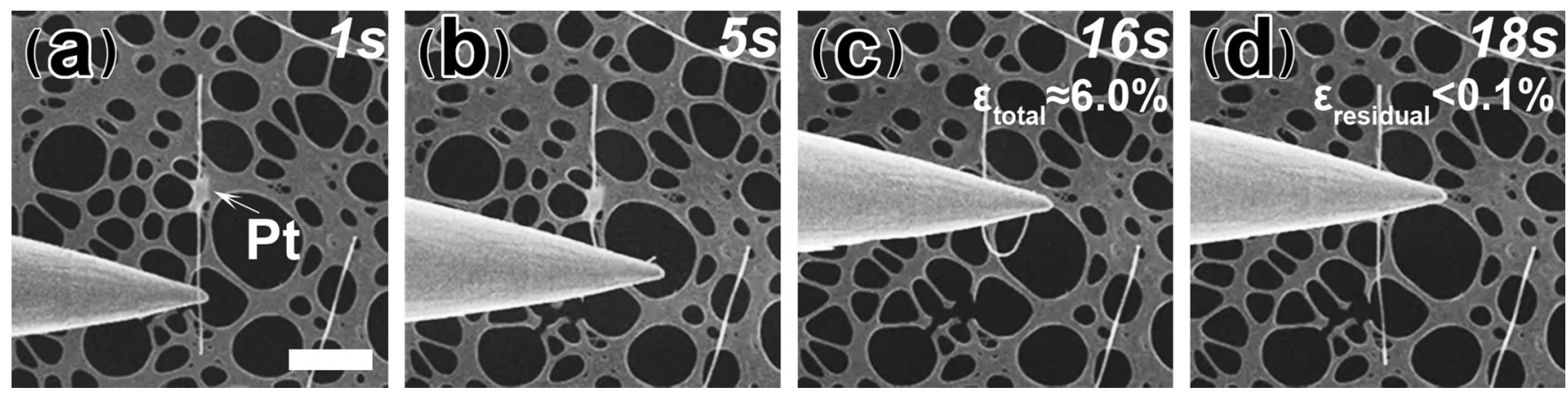

FIG. 1. Typical deformation process of GaAs NWs (diameter $\sim 50 \mathrm{~nm}$ ). The top right corner indicates the time-stamp (in seconds) for the image from the movie. The largest strain $\left(\varepsilon_{\text {total }} \sim 6 \%\right)$ occurred in $(\mathrm{c})$, and the NW recovered to close to the original shape (d) with very little residual plastic strain $(<0.1 \%)$. Scale bar: $2 \mu \mathrm{m}$

patches onto selected areas. Controllable strain can then be applied to a single NW with the tungsten manipulator and maintained on the TEM sample with deposition. The releasing of the strain can be done by breaking either the deposition or the surrounded supporting film. Note that the SEM stage had been adjusted in order to find the largest curvature radius to obtain an accurate total strain value.

A typical complete bending deformation process of a NW is shown by series of SEM images in Figures 1(a)-1(d). A twin-free GaAs NW with a diameter of $\sim 50 \mathrm{~nm}$ was affixed to a supporting carbon film on a copper grid by a small patch of platinum. A tungsten nano-manipulator was then used to progressively apply a torque to bend the NW in a controlled manner. The radius of the bending curvature, $R$, was measured in real-time and the total strain at edge, edge, was calculated using the formula $\varepsilon_{\text {total }}^{\text {edge }}=D /(D+2 R)$, where $D$ is the NW diameter. ${ }^{19,20}$ As marked in Figure 1(c), the largest strain reached $\sim 6 \%$ when the NW was bent almost $180^{\circ}$. When the external load was released, the NW nearly returned to its original shape, as shown in Figure 1(d), with very little residual strain $\left(\varepsilon_{\text {residual }}<0.1 \%\right)$. The finite residual strain is proof positive that plastic deformation was activated during the deformation process. It was also observed that the GaAs NWs were very fragile after exposure to the $6 \%$ strain and slight external turbulence, such as the gas flow from the platinum gas injection system, or movement of the micro-manipulator could trigger fracture at the region which was most deformed. Deformation can be completely released in our method, offering the capability to study atomic-scale structure before deformation, during deformation, and after release.

The evolution of deformation microstructure within the NW during the deformation-recovery process is presented in Figure 2. A NW with a diameter of $\sim 56.4 \mathrm{~nm}$ was deformed
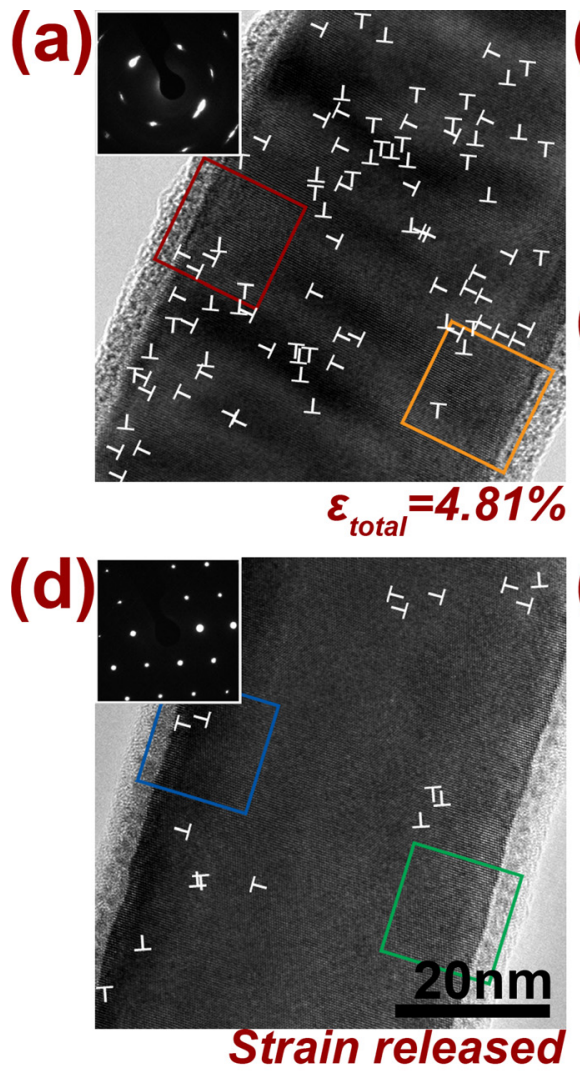
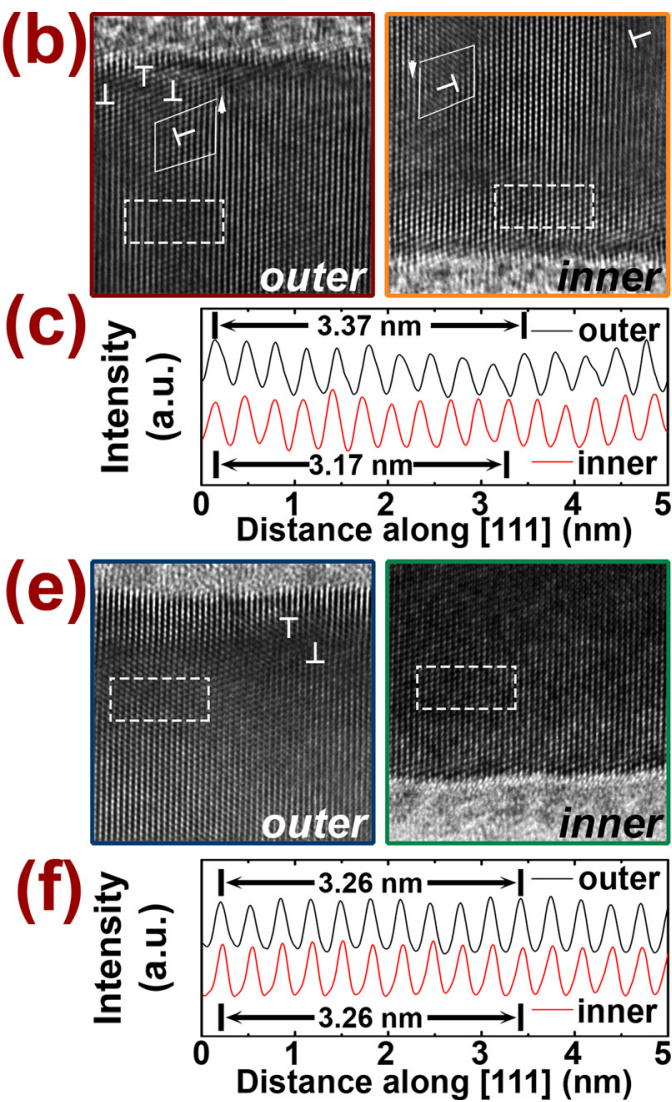

FIG. 2. Microstructural evolution of GaAs NWs following a complete deformation-recovery process. (a) HRTEM image and corresponding SAED pattern of a NW deformed $4.81 \%$. A very high density of dislocations is seen. (b) Enlarged from the regions indicated in (a), Burgers circuits were drawn to determine the dislocation type. (c) Line intensity profiles from dashed rectangles in (b) showing the distorted $\{111\}$ d-spacing under strain. (d) HRTEM image of the NW following removal of the applied load, indicating that the NWs had recovered spontaneously to something very close to the original shape, and that the dislocation density was reduced dramatically such that residual dislocations were found mostly near the edge of the NW. The inset SAED pattern is identical to that of the undeformed sample. (e) Enlarged regions from (d). (f) Line intensity profiles from dashed rectangles in (e) demonstrating the recovery of the bond stretching/compression. Labels $(\mathrm{T})$ indicate the dislocation lines in the HRTEM images. In (c) and (f), ten interplanar lattice distances have been marked. 
to a strain of $\sim 4.81 \%$, representing the case of high strain deformation. The HRTEM image in Figure 2(a) reveals a superposition of strain contrast and lattice fringe contrast. A selected-area electron diffraction (SAED) pattern along the $1 \overline{1} 0$ zone axis in the inset of Figure 2(a) reveals that the diffraction spots were elongated into arcs due to the continuous pure bending deformation of the NW. Careful examination of the crystal lattice fringes reveals an expansion at the outerside of the NW, and a compression at the inner-side, indicating the presence of elastic strain $\varepsilon_{\text {elastic }}$. The regions near the inner and outer edges of the NW are enlarged in Figure 2(b) and were used to measure the d-spacings of the $\{111\}$ planes along the NW axial direction. The line profiles extracted from Fig. 2(b) are displayed in Figure 2(c). The spacing for these $\{111\}$ planes deviated substantially from the standard (undeformed) value of $0.326 \mathrm{~nm}$. At least 20 regions near either the inner or outer edges at the most deformed regions were measured to obtain reliable average values for the inner and outer $\{111\}$ plane d-spacing, $d_{\text {inner }}$ and $d_{\text {outer }}$, respectively. These values were then used to calculate the elastic strain $\varepsilon_{\text {elastic }}$ caused by the lattice distortion using the equation: $\varepsilon_{\text {elastic }}=\left(d_{\text {outer }}-d_{\text {inner }}\right) /\left(d_{\text {outer }}+d_{\text {inner }}\right) .{ }^{12}$ This calculation revealed a key insight into the deformation mechanism: the calculated elastic strain $\varepsilon_{\text {elastic }}$ was $\sim 3.06 \%$, which is significantly smaller than the effective total strain at $\mathrm{d}$-spacing measurement regions, $\varepsilon_{\text {total }}^{d} \sim 3.61 \%$. The difference must be made up by plastic deformation mediated by discrete dislocation activity, displacive deformation twinning, or stress-induced phase transformations. ${ }^{4}$

In fact, dislocations were observed extensively across the deformed GaAs NW, as labelled $(\perp)$ in Figure 2(a), and this is clear evidence of dislocation-based deformation. Detailed Burgers circuit analysis (Figure 2(b)) indicated that the dislocations were all $1 / 2\langle 110\rangle$ type perfect dislocations. No nanotwins, extended dislocation/stacking faults, or phase transformations were observed in the deformed NWs. It has been reported that the nucleation and gliding of perfect dislocations is responsible for plastic deformation in bulk $\mathrm{GaAs}^{21,22}$ and we propose that similar mechanisms dominate in the present work. Therefore, the difference between total strain and elastic stain made up by dislocation-based plastic strain $\varepsilon_{\text {plastic }}$ was determined by $\varepsilon_{\text {plastic }}=\varepsilon_{\text {total }}^{d}-\varepsilon_{\text {elastic }}$. Hence, $\varepsilon_{\text {plastic }}$ is $\sim 0.6 \%$ for the NW when $\varepsilon_{\text {total }}^{d} \sim 3.61 \%$. Therefore, remarkably, Figure 2 provides direct evidence for the parallel development of lattice- and dislocation-based deformation.

Figure 2(d) is a HRTEM image of the NW microstructure after the stress was released. The dislocation density was observed to dramatically reduce, such that only a low density of dislocations close to the edge region of the NW remained, which is believed to be responsible for the small residual strain $\varepsilon_{\text {residual }}(<0.1 \%)$ in the NW. The inset SAED pattern reveals that the diffraction arcs transformed back to diffraction spots and our HRTEM images, such as those provided in Figure 2(e), reveal that the crystal was restored to high quality. Measurements of the $\{111\}$ planar spacing, illustrated by the line profile in Figure 2(f), confirmed that the elastic deformation had fully recovered. Surprisingly, the data indicate that the dislocation-based deformation has almost completely recovered spontaneously, resembling an elastic deformation process.

To further investigate the behaviour and interaction between the elastic and plastic deformation, a number of NWs were deformed to different strain levels. The structural information was quantified and summarized in Table I. The proportions of elastic and plastic strain components as a function of total strain were presented in Figure 3. It is evident and striking that plastic strain $\varepsilon_{\text {plastic }}$ develops in parallel with elastic strain $\varepsilon_{\text {elastic }}$ throughout the whole deformation range. ${ }^{23}$ It is in sharp contrast to the conventional theory that dislocation-based deformation is plastic and irreversible, ${ }^{1}$ and it is also in direct contradiction to the previous reports that NWs are free of dislocations within the elastic regime. ${ }^{24}$

We propose that the nanoscale dimensions of the NWs and the bending deformation mode are responsible for the simultaneous development of significant elastic and plastic components of deformation from very low strains through to fracture. The NW surface provides abundant sources for dislocation nucleation due to the high surface to volume ratio in these nanostructured materials, and this makes it possible to produce a very high density of dislocations during deformation. The nucleation of dislocations at the surface or at grain boundaries usually requires much lower stress than the selfmultiplication of dislocations by, e.g., Frank-Read sources in nanocrystalline materials. ${ }^{25,26}$ For bending deformation of a

TABLE I. Summary of the microstructures and strains of NWs that have been deformed to or released from different strain levels.

\begin{tabular}{|c|c|c|c|c|c|c|c|}
\hline \multirow[b]{2}{*}{$\begin{array}{l}\text { Total strain } \\
\text { at edge } \varepsilon_{\text {total }}^{\text {edge }}(\%)\end{array}$} & \multirow[b]{2}{*}{$\begin{array}{c}\text { Total strain } \\
\text { at } d_{\text {outer }}, d_{\text {inner }} \varepsilon_{\text {total }}^{d}(\%)\end{array}$} & \multirow[b]{2}{*}{$\begin{array}{c}\text { Elastic strain } \\
\varepsilon_{\text {elastic }}(\%)\end{array}$} & \multirow[b]{2}{*}{$\begin{array}{c}\text { Plastic } \\
\text { strain } \varepsilon_{\text {plastic }}(\%)\end{array}$} & \multicolumn{2}{|c|}{ (111) d-spacing (nm) } & \multirow[b]{2}{*}{$\begin{array}{l}\text { Amount of dislocations/area } \\
\text { of measurement }\left(\mathrm{nm}^{2}\right)\end{array}$} & \multirow[b]{2}{*}{$\begin{array}{c}\text { Dislocation } \\
\text { density } \rho\left(10^{16} \mathrm{~m}^{-2}\right)\end{array}$} \\
\hline & & & & $\begin{array}{l}\text { Outer } \\
d_{\text {outer }}\end{array}$ & $\begin{array}{l}\text { Inner } \\
d_{\text {inner }}\end{array}$ & & \\
\hline 1.50 & 1.13 & 0.79 & 0.34 & 0.3286 & 0.3234 & $21 / 2909.41$ & 0.72 \\
\hline 2.02 & 1.52 & 1.08 & 0.44 & 0.3293 & 0.322 & $29 / 2786.15$ & 1.04 \\
\hline 2.68 & 2.01 & 1.48 & 0.53 & 0.3292 & 0.3196 & $43 / 3091.46$ & 1.39 \\
\hline 3.23 & 2.42 & 1.83 & 0.59 & 0.332 & 0.3201 & $45 / 2874.24$ & 1.57 \\
\hline 3.88 & 2.91 & 2.36 & 0.55 & 0.3347 & 0.3193 & $64 / 2793.54$ & 2.29 \\
\hline 4.46 & 3.34 & 2.86 & 0.48 & 0.3366 & 0.3178 & $104 / 3158.81$ & 3.29 \\
\hline 4.81 & 3.61 & 3.06 & 0.55 & 0.3372 & 0.3174 & $106 / 2892.21$ & 3.67 \\
\hline 5.53 & 4.15 & 3.16 & 0.99 & 0.3376 & 0.3169 & $180 / 2980.54$ & 6.04 \\
\hline Released from 2.02 & $<0.1 \%$ & $<0.1 \%$ & $<0.1 \%$ & 0.3261 & 0.326 & $21 / 2853.69$ & 0.74 \\
\hline Released from 4.81 & $<0.1 \%$ & $<0.1 \%$ & $<0.1 \%$ & 0.3261 & 0.3259 & $11 / 2794.51$ & 0.39 \\
\hline
\end{tabular}




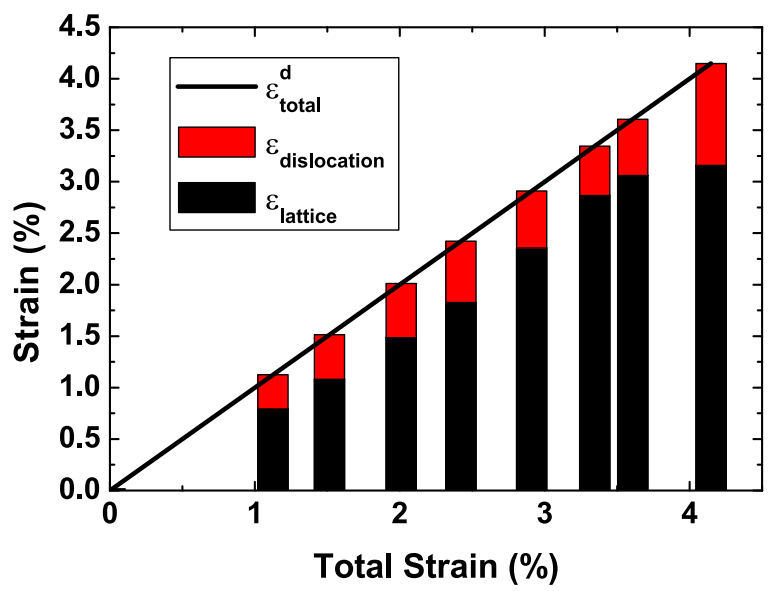

FIG. 3. Elastic and plastic strain components with respect to the total strain, illustrating that elastic deformation and plastic deformation occur simultaneously.

NW, the strain type changes from tension at one side to compression at the other. The magnitude of strain gradually decreases from the edge to centre, so that the stress minimizes somewhere around the geometric axial centre of the NW. Such a low stress area acts as a barrier to prevent dislocations from passing through the whole NW. Therefore, dislocations generated on the crystalline surface and by multiplication at regions with large strains build up rapidly in the NW, reaching an extremely high dislocation density on the order of $10^{16} \mathrm{~m}^{-2}$, which is nine orders of magnitude higher than those usually observed in high-quality bulk GaAs single crystals $\left(10^{5} \mathrm{~m}^{-2}\right){ }^{27}$ Consequently, very significant work hardening occurs because of the repulsive force between dislocations with the same Burgers vectors and the tangling of dislocations that glide on different slip systems. This leads to a very rapid increase in the flow stress during deformation, which is distinctive from conventional deformation in coarse-grained materials where the increase in flow stress is limited. The dramatic increase in flow stress results in further increases in significant elastic strain after the initiation of plastic deformation.

When the applied stress was removed, the large internal stress was released through the recovery of elastic strain. The internal stress is opposite in direction and approximately equivalent in magnitude to the applied stress during the initial recovery process. This internal stress spontaneously activated and drove the dislocation motion backwards. It is known that surfaces or grain boundaries of nanomaterials act as both the source and sink of dislocations. ${ }^{26,28}$ Hence, the reverse motion of the dislocations led to the reversal of the plasticity, which has been described by molecular dynamics simulations. ${ }^{14}$ In this study, it was shown that, at the point whereby most of the dislocations had been annihilated at the surface, the plastic strain was largely recovered, and so the process resembled that of the recovery of elastic deformation. ${ }^{29}$

In summary, we have discovered the simultaneous operation of super elastic and dislocation-based plastic strain during the bending deformation process of GaAs NWs, which is in sharp contrast to deformation theory. It indicates that the observed super-recoverable deformation ${ }^{10-17}$ is neither simply elastic nor reversibly plastic in nature, but the coupling of both modes. In future, strain-stress curves need to be measured, at the same time of the atomic-scale observation.

This work was supported in part by the Australian Research Council. The authors acknowledge the facilities and technical assistance from staff at the Australian Microscopy \& Microanalysis Research Facility node (Sydney Microscopy \& Microanalysis) at the University of Sydney. Growth facilities used in this work were supported by the Australian National Fabrication Facility (ANFF) at the Australian National University.

${ }^{1}$ J. Lubliner, Plasticity Theory (DOVER Publication, Inc., 2008).

${ }^{2}$ J. Li, K. J. Van Vliet, T. Zhu, S. Yip, and S. Suresh, Nature 418(6895), 307 (2002).

${ }^{3}$ Y. Champion, C. Langlois, S. Guérin-Mailly, P. Langlois, J.-L. Bonnentien, and M. J. Hÿtch, Science 300(5617), 310 (2003).

${ }^{4}$ L. Wang, P. Liu, P. Guan, M. Yang, J. Sun, Y. Cheng, A. Hirata, Z. Zhang, E. Ma, M. Chen, and X. Han, Nat. Commun 4, 2413 (2013).

${ }^{5}$ N. W. Moore, J. Luo, J. Y. Huang, S. X. Mao, and J. E. Houston, Nano Lett. 9(6), 2295 (2009).

${ }^{6}$ W. D. Callister and D. G. Rethwisch, Materials Science and Engineering: An Introduction, 6th ed. (Wiley India Pvt. Limited, 2009).

${ }^{7}$ B. Wu, A. Heidelberg, and J. J. Boland, Nature Mater. 4(7), 525 (2005).

${ }^{8}$ M.-Feng Yu, B. S. Files, S. Arepalli, and R. S. Ruoff, Phys. Rev. Lett. 84(24), 5552 (2000).

${ }^{9}$ C. Q. Chen and J. Zhu, Appl. Phys. Lett. 90(4), 043105 (2007).

${ }^{10}$ X. D. Han, K. Zheng, Y. F. Zhang, X. N. Zhang, Z. Zhang, and Z. L. Wang, Adv. Mater. 19(16), 2112 (2007).

${ }^{11}$ X. D. Han, Y. F. Zhang, K. Zheng, X. N. Zhang, Z. Zhang, Y. J. Hao, X. Y. Guo, J. Yuan, and Z. L. Wang, Nano Lett. 7(2), 452 (2007).

${ }^{12}$ X. Han, L. Kou, X. Lang, J. Xia, N. Wang, R. Qin, J. Lu, J. Xu, Z. Liao, X. Zhang, X. Shan, X. Song, J. Gao, W. Guo, and D. Yu, Adv. Mater. 21(48), 4937 (2009).

${ }^{13}$ Y.-B. Wang, L.-F. Wang, H. J. Joyce, Q. Gao, X.-Z. Liao, Y.-W. Mai, H. H. Tan, J. Zou, S. P. Ringer, H.-J. Gao, and C. Jagadish, Adv. Mater. 23(11), 1356 (2011).

${ }^{14}$ X. Li, Y. Wei, W. Yang, and H. Gao, Proc. Natl. Acad. Sci. U.S.A. 106(38), 16108 (2009).

${ }^{15}$ G. Ziegenhain and H. M. Urbassek, Philos. Mag. Lett. 89(11), 717 (2009).

${ }^{16}$ M. W. Barsoum, T. Zhen, S. R. Kalidindi, M. Radovic, and A. Murugaiah, Nature Mater. 2(2), 107 (2003).

${ }^{17}$ E. Demir and D. Raabe, Acta Mater. 58(18), 6055 (2010).

${ }^{18}$ H. J. Joyce, Q. Gao, H. Hoe Tan, C. Jagadish, Y. Kim, X. Zhang, Y. Guo, and J. Zou, Nano Lett. 7(4), 921 (2007).

${ }^{19}$ L. L. D. Landau, E. M. Lifshits, A. M. Kosevitch, and L. P. Pitaevskiı̌, Theory of Elasticity (Butterworth-Heinemann, 1986), Vol. 7.

${ }^{20}$ L. Wang, K. Zheng, Z. Zhang, and X. Han, Nano Lett. 11(6), 2382 (2011).

${ }^{21}$ T. Suzki, T. Yasutomi, T. Tokuoka, and I. Yonenaga, Philos. Mag. A 79(11), 2637 (1999).

${ }^{22}$ S. Wang and P. Pirouz, Acta Mater. 55(16), 5500 (2007).

${ }^{23}$ C. R. Weinberger and W. Cai, J. Mater. Chem. 22(8), 3277 (2012).

${ }^{24}$ K. Zheng, X. Han, L. Wang, Y. Zhang, Y. Yue, Yan Qin, X. Zhang, and Z. Zhang, Nano Lett. 9(6), 2471 (2009).

${ }^{25} \mathrm{H}$. Van Swygenhoven, Science 296(5565), 66 (2002).

${ }^{26}$ X. Z. Liao, Y. H. Zhao, S. G. Srinivasan, Y. T. Zhu, R. Z. Valiev, and D. V. Gunderov, Appl. Phys. Lett. 84(4), 592 (2004).

${ }^{27}$ P. Rudolph, Cryst. Res. Technol. 40(1-2), 7 (2005).

${ }^{28}$ Z. Budrovic, H. Van Swygenhoven, P. M. Derlet, S. Van Petegem, and B. Schmitt, Science 304(5668), 273 (2004).

${ }^{29}$ J. Rajagopalan, J. H. Han, M. Taher, and A. Saif, Science 315(5820), 1831 (2007). 